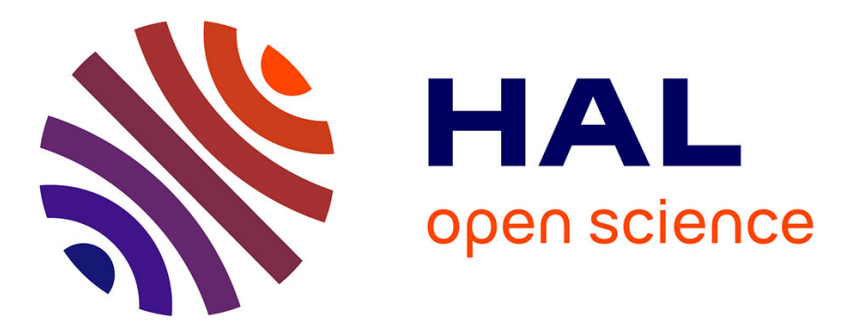

\title{
Optical properties of cometary particles collected by COSIMA: Assessing the differences between microscopic and macroscopic scales
}

Y Langevin, S Merouane, M Hilchenbach, M Vincendon, K Hornung, C Engrand, R Schulz, J Kissel, J Ryno

\section{To cite this version:}

Y Langevin, S Merouane, M Hilchenbach, M Vincendon, K Hornung, et al.. Optical properties of cometary particles collected by COSIMA: Assessing the differences between microscopic and macroscopic scales. Planetary and Space Science, 2019, 182 (104815), 10.1016/j.pss.2019.104815 . hal03051884

\section{HAL Id: hal-03051884 \\ https://hal.science/hal-03051884}

Submitted on 21 Dec 2020

HAL is a multi-disciplinary open access archive for the deposit and dissemination of scientific research documents, whether they are published or not. The documents may come from teaching and research institutions in France or abroad, or from public or private research centers.
L'archive ouverte pluridisciplinaire HAL, est destinée au dépôt et à la diffusion de documents scientifiques de niveau recherche, publiés ou non, émanant des établissements d'enseignement et de recherche français ou étrangers, des laboratoires publics ou privés. 


\title{
Optical properties of cometary particles collected by COSIMA: Assessing the differences between microscopic and macroscopic scales
}

\author{
Y. Langevin ${ }^{\mathrm{a}, *}$, S. Merouane ${ }^{\mathrm{b}}$, M. Hilchenbach ${ }^{\mathrm{b}}$, M. Vincendon ${ }^{\mathrm{a}}$, K. Hornung ${ }^{\mathrm{c}}$, C. Engrand ${ }^{\mathrm{d}}$, \\ R. Schulz ${ }^{\mathrm{e}}$, J. Kissel ${ }^{\mathrm{b}}$, J. Ryno ${ }^{\mathrm{f}}$ \\ ${ }^{a}$ Institut d'Astrophysique Spatiale, CNRS/Univ. Paris Saclay, Orsay, France \\ ${ }^{\mathrm{b}}$ Max-Planck Institut für Sonnensystemforschung, Göttingen, Germany \\ ${ }^{\text {c } U n i v e r s i t a ̈ t ~ d e r ~ B u n d e s w e h r, ~ N e u b i b e r g, ~ G e r m a n y ~}$ \\ d CSNSM, CNRS/Univ. Paris-Sud, Orsay, France \\ ${ }^{\mathrm{e}}$ European Space Agency Scientific Support Office, Noordwijk, the Netherlands \\ ${ }^{\mathrm{f}}$ Finnish Meteorological Institute, Helsinki, Finland
}

\section{A R T I C L E I N F O}

\section{Keywords:}

Comets

Dust

\begin{abstract}
A B S T R A C T
The COSIMA mass spectrometer on-board Rosetta was equipped with an optical microscope, Cosiscope, which identified several 10,000 cometary particles collected on targets exposed during the orbital phase around the nucleus of comet 67P Churyumov-Gerasimenko. The median value of reflectance factors evaluated from Cosiscope images for large collected particles ( $~ 10.5 \%$ (Langevin et al., 2017), lies above the range of reflectance observed by the OSIRIS camera at a similar wavelength (5-7\%, (Fornasier et al., 2015), but at much larger scales (a few 10 $\mathrm{cm}$ instead of a few $10 \mu \mathrm{m}$ ). In order to better understand this discrepancy, the assumptions underlying the derivation of reflectance factors have been reassessed using laboratory measurements of COSIMA targets and analogs of cometary particles. The approach of Langevin et al. is validated, but we consider that the uncertainty on reflectance factors was conservative. The reflectance factors are likely to lie in the lower half of the previously estimated range, which reduces (but does not eliminate) the discrepancy with OSIRIS albedos. The remaining discrepancy can be attributed primarily to the difference in scale (factor 10,000 in pixel size between COSIMA and OSIRIS):
\end{abstract}

\section{Introduction}

The COSIMA mass spectrometer on board the ROSETTA spacecraft (Kissel et al., 2007) analyzed cometary particles collected on sets ("target assemblies") of 3 targets $10 \mathrm{~mm} \times 10 \mathrm{~mm}$ in size exposed simultaneously to the cometary environment. 72 targets where available on 24 target assemblies which could be picked-up by a grasping device for exposure. COSIMA was equipped with a microscope (Cosiscope) for imaging targets before and after exposure so as to detect collected particles (Langevin et al., 2016). Grazing incidence lighting by either one or the other of two red LED's ( $\lambda=640 \mathrm{~nm}$ ) positioned left and right of the targets (Fig. 1) was selected so as to maximize the detection capability on smooth "gold black" targets (Hornung et al., 2016) which constituted half of the targets selected for flight. Targets with a rougher surface ("silver black", "palladium", "platinum") were not well suited for optical detection, but they were included in case "gold black" targets turned out to have low collection efficiency for cometary particles. The first target assembly to be exposed, from orbit insertion around the nucleus of 67P/Churyumov-Gerasimenko (early August 2014) to December 2014, was equipped with 3 "gold black" targets for optimum optical detection performance as low fluxes of small cometary particles were expected at heliocentric distances beyond $3 \mathrm{AU}$. The outcome of the collection and detection scheme on the first 3 exposed targets was far beyond expectations, as more than 2000 collected particles (or fragments of particles) were identified including 50 particles larger than $100 \mu \mathrm{m}$ in size. As the "gold black" targets of the first exposed targets proved quite successful in collecting cometary grains, the 6 target assemblies exposed from end of December 2014 to September 2016 (end of the orbital phase) were also selected among those holding mainly gold black targets, so that 17 of the 21 targets exposed during the orbital phase were "gold black" targets. Taking into account fragmentation events, the 35,000 particles or fragments of particles which have been identified on the exposed targets correspond to

\footnotetext{
* Corresponding author.

E-mail address: yves.langevin@ias.u-psud.fr (Y. Langevin).
} 


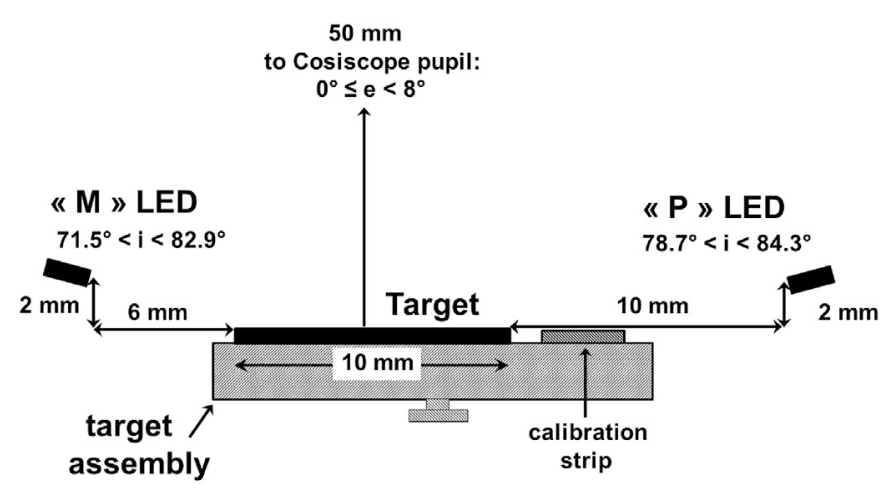

Fig. 1. observation geometry for Cosiscope. Three square targets $10 \times 10 \mathrm{~mm}$ across are exposed side by side on a target assembly. They are imaged by placing each target in front of a camera with an entrance pupil $50 \mathrm{~mm}$ away from the target, so that the emergence ranges from $0^{\circ}$ at the center of the target to $8^{\circ}$ at the corners. The target is illuminated by either one or the other of two LED's positioned $2 \mathrm{~mm}$ above the target plane (M LED to the left, P LED to the right), with an asymmetrical set up due to the calibration strip on one side of the three targets. The incidence along the center line increases from $71.5^{\circ}$ at the left edge of the target to $82.9^{\circ}$ at the right edge for the M LED. It decreases from $84.3^{\circ}$ to $78.7^{\circ}$ for the $\mathrm{P}$ LED. The phase remains close to $80^{\circ}$, ranging from 77.2 to $79.7^{\circ}$ along the center line for the M LED and from $78.6^{\circ}$ to $84.4^{\circ}$ for the P LED.

1200-1600 parent particles (Merouane et al., 2017). The collection of several hundred large particles made it possible to obtain resolved images and to address the typology of cometary particles, dominated by aggregates (Langevin et al., 2016).

Cosiscope images are obtained at high incidence $\left(71.5^{\circ}-85^{\circ}\right)$, small emergence $\left(<8^{\circ}\right)$ and a phase close to $80^{\circ}$ (Fig. 1). This grazing incidence lighting scheme which proved very effective for detection is not optimum for determining the optical properties of collected particles, as light levels are primarily controlled by their topography, contrary to low incidence measurements were light levels are primarily controlled by reflectance. Nevertheless, it was possible to constrain the reflectance factor of collected particles (Langevin et al., 2017) by comparing the maximum representative light level from a given particle to that at the same location on a "silver black" target (reflectance factor $\sim 11.8 \%$ ). The signal from a "gold black" target (reflectance factor $\sim 1.8 \%$ at low incidence) is very low, hence using this type of target as a reference would have resulted in very large uncertainties. One has to make an assumption on the minimum incidence (considered to be $\sim 45^{\circ}$ ), hence there are large error bars. As indicated in (Langevin et al., 2017); the $45^{\circ}$ assumption can result in an overestimation of the reflectance factor by at most a factor 1.4 ( $\mathrm{i}=0^{\circ}$ instead of $45^{\circ}$ ). The mean slope on the side facing the LED can be determined from the length of the shadow providing the height of the particle above the substrate. It is $>25^{\circ}$ for nearly all large particles, resulting in a mean incidence lower than $60^{\circ}$ for a phase of $84.4^{\circ}$ (maximum phase for the "P" LED), even lower for particles closer to the LED. The minimum incidence cannot be higher than the mean incidence along the side facing the LED, hence the possible underestimation of the reflectance factor cannot exceed $1.4\left(\mathrm{i}=60^{\circ}\right.$ instead of $\left.45^{\circ}\right)$.

This approach provided a range of reflectance factors from $3 \%$ to $20 \%$ for particles collected by COSIMA, with a median reflectance factor of $10.5 \%$. OSIRIS measured the photometric properties of the nucleus of 67P/Churyumov-Gerasimenko at macroscopic scales, from a few $10 \mathrm{~cm}$ up (Fornasier et al., 2015; Feller et al., 2016). At $645 \mathrm{~nm}$ (a wavelength very close to that of the Cosiscope LED's), the reflectance at $0^{\circ}$ phase angle ranges from 5 to $7 \%$. Therefore, the reflectance at macroscopic scales appears to be significantly lower than that estimated for $100 \mu \mathrm{m}$ particles.

In this article, we revisit the assumptions made when evaluating reflectance factors with Cosiscope, so as to evaluate the possible causes for the discrepancy. One of the possible issues deals with the evolution of radiance with incidence. The 2017 article (Langevin et al., 2017) assumed a Lambert scattering behavior, with a radiance proportional to $\cos (\mathrm{i})$. The OSIRIS team uses a different relationship between radiance, emergence and incidence (Lommel-Seeliger). Section 2 is dedicated to laboratory experiments exploring the evolution of radiance with incidence at low emergence $\left(<10^{\circ}\right.$ for Cosiscope images of particles) for the "silver black" targets used as reference for the evaluation of reflectance factors and for different types of analog cometary particles. The conservative range of reflectance factors in (Langevin et al., 2017) (factor 0.7 to 1.4 compared to that which would result from a $45^{\circ}$ incidence) will also be reevaluated. This leads to lower most probable reflectances for COSIMA particles, but they are still higher than the range of reflectances observed by OSIRIS at macroscopic scales.

In section 3 , we discuss the possible causes of the remaining differences between the macroscopic photometric properties observed by OSIRIS and that evaluated for cometary particles collected by COSIMA at scales of a few $10 \mu \mathrm{m}$.

\section{Lommel-Seeliger scattering versus Lambert scattering: validity domains and experimental evidence from COSIMA targets and cometary analogs}

The nucleus of 67P/Churyumov-Gerasimenko is one of the darkest solar system bodies observed up to now at close range (Fornasier et al., 2015), and particles collected by COSIMA are also quite dark (median albedo 0.105, (Langevin et al., 2017). There are basically two ways a surface can be dark: if the single scattering albedo is low, the photons are absorbed at the first interaction. If the surface is highly porous (with an opaque dust cloud as an extreme case), multiple scattering results in an apparent albedo which can be much lower than the single scattering albedo.

The Lommel-Seeliger law is used for comparing different optical geometries by the OSIRIS team (Fornasier et al., 2015; Feller et al., 2016). It is based on a single scattering assumption with isotropic scattering (no forward or backward enhanced scattering), and it provides an adequate first estimate for the surfaces of most solar system bodies. The radiance is proportional to $\omega_{0}$ (the single scattering albedo) and to $\cos (\mathrm{i}) /(\cos (\mathrm{i})+$ $\cos (\mathrm{e}))$ where " $\mathrm{i}$ " is the incidence and " $\mathrm{e}$ " is the emergence. For the low emergences $\left(<10^{\circ}\right)$ of Cosiscope, the ratio between light levels observed at a given incidence and that at incidence $0^{\circ}$ is:

$\mathrm{R}(\mathrm{i}) / \mathrm{R}\left(0^{\circ}\right)=2 \cos (\mathrm{i}) /(1+\cos (\mathrm{i}))$

Lambert scattering applies when multiple scattering dominates. This weakens the connection between the incoming and outgoing directions, so that the radiance does not markedly depend on emergence, with an apparent albedo which is much lower than the single scattering albedo. Radiance is then simply proportional to $\cos (\mathrm{i})$ :

$\mathrm{R}(\mathrm{i}) / \mathrm{R}\left(0^{\circ}\right)=\cos (\mathrm{i})$

Multiple scattering definitely dominates for aerosols constituted by mineral dust, which have a very high scattering albedo in the visible (>0.9, (Wolff et al., 2009; Moosmüller et al., 2012), as the albedo of Mars dust storms with high optical thicknesses is only $40-45 \%$ (Wolff et al., 1999). A similar albedo is observed for dust deposits on the surface of Mars, so that multiple scattering must also be involved. Monte-Carlo modelling has shown that for aerosols and highly porous media the Lambert scattering law applies at low emergence even with Henley-Greenstein distributions favoring forward or backward scattering (Vincendon et al., 2007).

Comparing with the photometric results of OSIRIS requires retracing the observed radiance to a reference photometric geometry with normal incidence and emergence. All the collected cometary particles have been observed by Cosiscope on target areas with incidences from $70^{\circ}$ to $85^{\circ}$, while an incidence of $45^{\circ}$ is assumed for the surface element of the particle facing the LED which corresponds to the maximum representative light level (Langevin et al., 2017). The radiance ratio between 
normal incidence and the observed incidence is larger with Lambert (2) than with Lommel-Seeliger (1) by a factor $2 /(1+\cos (\mathrm{i}))$, from 1.17 at $45^{\circ}$ to 1.5 at $70^{\circ}$ and 1.84 at $85^{\circ}$ (Langevin et al., 2017). determines reflectance factors by comparing the maximum radiance to that of a reference target at the same location. This means that if either the particle or the target follows a Lommel-Seeliger law instead of a Lambert law, the resulting reflectance factor of the particle at low incidence has to be reevaluated:

- Lommel-Seeliger target, Lambert particle: increase by a factor 1.5 $\left(70^{\circ}\right)$ to $1.84\left(85^{\circ}\right)$

- Lambert target, Lommel-Seeliger particle: decrease by a factor 1.17.

- Lommel-Seeliger target and particle: increase by a factor $1.28\left(70^{\circ}\right)$ to $1.57\left(85^{\circ}\right)$

As can be seen, only one hypothesis (Lambert target, Lommel-Seeliger particle) results in lower reflectance factors at $0^{\circ}$ incidence. The other two would increase the discrepancy with OSIRIS results by a very significant factor.

Laboratory experiments have been performed at MPS Göttingen with "silver black" targets and particles so as to provide constraints on the photometric model. The experimental set-up is shown on Fig. 2 (left). In this set-up, the camera is fixed, located on top of the target. The LED $(\lambda=$ $640 \mathrm{~nm}$, the same as Cosiscope LED's) is placed on a movable arm in order to change the incidence angle on the target. Due to mechanical limitations, the maximum incidence that could be reached is $70^{\circ}$, corresponding to the lower end of the range of incidences relevant for Cosiscope.

Two different materials, used as analogs of cometary dust particles, were measured using the sample set-up (Fig. 2, right). The first analogs used are aggregates of $\mathrm{SiO}_{2}$ impacted on a COSIMA "gold black" target at low velocity (Ellerbroek et al., 2017). The collected particles exhibit a variety of shapes, from flattish to pyramidal. These different shapes are used to obtain evaluations of the reflectance at different incidence and emergence angles (Fig. 2, left). The second analog for consistency dust particles that have been used is a powder from the Allende carbonaceous chondrite deposited on a gold black target, which is more representative of cometary dust in terms of composition than the $\mathrm{SiO}_{2}$ aggregates. The results are shown in Fig. 3.

As can be seen from Fig. 3 the dispersion of measurements is large, in particular for small particles. However, the median value at each incidence is close to a Lambert relationship for the "silver black" target, while a Lommel-Seeliger relationship can be excluded. For $\mathrm{SiO}_{2}$ particles (which are brighter than typical cometary grains), most of the measurements lie below the values expected with a Lambert relationship. For meteoritical particles, the values at high incidence angle are close to the values expected for a Lambert scattering. These results provide supportive evidence for the Lambert assumption made by (Langevin et al., 2017).

This is not surprising for the targets as the low albedo of "metal black" comes from a very high porosity leading to a major role of multiple scattering. Most of the large collected particles for which the reflectance factor has been evaluated are aggregates. Even "compact" particles with a well-defined outer surface exhibit sub-structure, and many of the largest compact particles have disintegrated when analyzed due to electrostatic effects (Hilchenbach et al., 2017). Cometary particles are therefore very weak (Hornung, 2014), in line with macroscopic evaluations of the tensile strength of overhangs on the nucleus (3-15 Pa, Groussin et al., 2015). The radiance profiles across the particles shows that the mean free path of photons within the particle, $20-25 \mu \mathrm{m}$ (Langevin et al., 2017), is much larger than the wavelength $(0.64 \mu \mathrm{m})$. Cometary particles at a scale of a few $\mu \mathrm{m}$ collected by MIDAS are also aggregates (Bentley et al., 2016), and dust aggregates have been observed by OSIRIS close to the Rosetta spacecraft (Güttler et al., 2017). Therefore, light scattering by cometary particles does not primarily occur as a single scattering event at a well-defined outer interface, but through interaction with scattering centers within the particle. As will be discussed later on, the meaning of photometric parameters such as incidence needs to be carefully reassessed in such cases.

An effect which could play a role for the "silver black" targets at incidences beyond $70^{\circ}$ is self-shadowing. While much smoother than the simulated "primitive body surface" of (Vincent, 2019); a "silver black" target exhibits more topography than "gold black" targets, which are very smooth. Self-shadowing has not a major impact if Lambert scattering applies, as the relevant parameter is the mean flux over an area, which decreases as $\cos (\mathrm{i})$ where " $\mathrm{i}$ " is the mean incidence, even if lighting is inhomogeneous at scales smaller than that at which the reflectance is evaluated. One cannot however exclude that self-shadowing could bring the light level below the Lambert assumption for very high incidences (hence extended shadows), resulting in overestimated reflectance factors for the particles.

\subsection{A reevaluation of the relevant range of incidences for the brightest representative pixels}

The range of incidences selected for bright representative pixels in (Langevin et al., 2017) on the slope facing the LED was 0.5-1, corresponding to incidences of $60^{\circ}-0^{\circ}$. This guaranteed that the actual
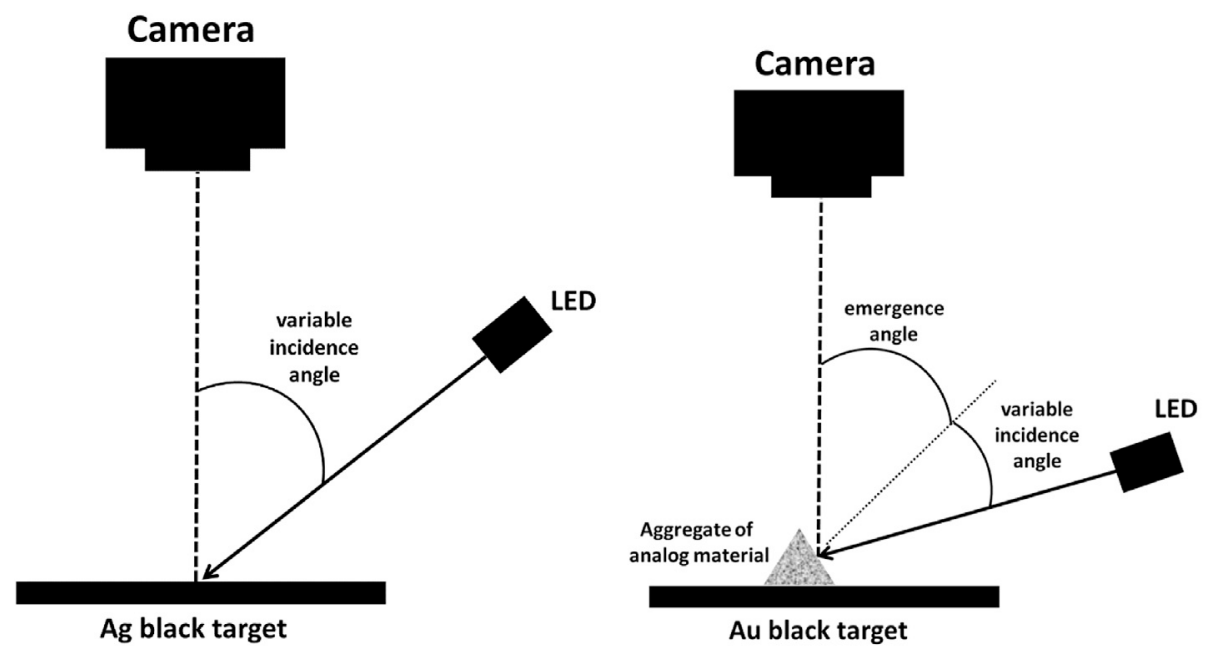

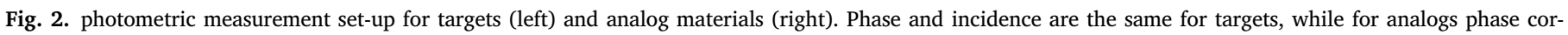
responds to the sum of emergence (typically $20-70^{\circ}$ ) and incidence, similarly to particles collected by COSIMA, observed at phase angles from $80^{\circ}$ to $85^{\circ}$. 

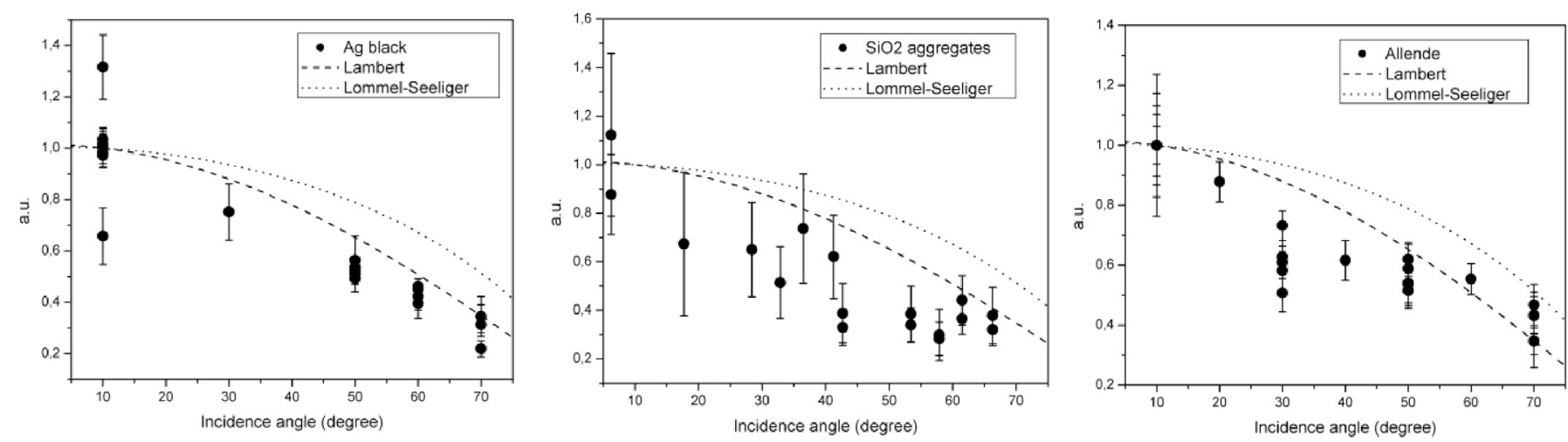

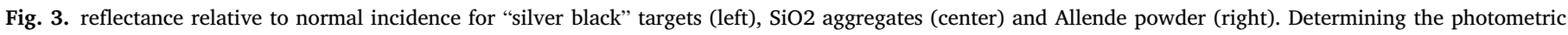

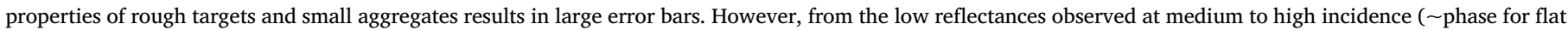
targets), a Lambert law is a better fit than the Lommel-Sieliger law used at macroscopic scale.

reflectance factor was at most a factor of 1.41 away from that evaluated assuming an incidence of $45^{\circ}(\cos (\mathrm{i})=0.714)$. This range was derived from the mean slope $\left(\sim 25^{\circ}\right.$ for most particles) corresponding to a $60^{\circ}$ mean incidence on the particle to be compared with an incidence of $85^{\circ}$ on the target. This is a conservative approach as bright representative pixels correspond to an optimum in terms of illumination combined with local reflectance, so that the local incidence for such pixels is expected to be lower than the mean incidence.

Most compact particles with well-defined outer surfaces present overhangs, so that the incidence profile goes down to $0^{\circ}$ (then back up to $5^{\circ}-15^{\circ}$ depending on the incidence on the local target area) close to the edge of the particle facing the LED. Even for the smallest such particles (100 $\mu \mathrm{m}$ across), a circular cross-cut would lead to a $20 \mu \mathrm{m}$ extent as seen from the direction of the camera for the area illuminated at incidences lower than $25^{\circ}(\cos (i)=0.9)$, larger than one pixel $(14 \mu \mathrm{m})$. A lower limit of 0.9 for $\cos (i)$, instead of 0.5 is therefore legitimate for these particles.

Aggregates are very irregular. "Rubble piles" and "shattered clusters" (Langevin et al., 2016) present components with well-defined boundaries, hence facets at the pixel scale $(14 \mu \mathrm{m})$ with low incidence. Furthermore, the mean free path $(20-25 \mu \mathrm{m})$ is in the same range as the size of such components (down to $25-50 \mu \mathrm{m}$ ). Whatever the incidence of the facet facing the camera, the radiance depends on the proportion of monomers within the pixel which are lit by the LED, including from the side of the particle facing the LED. There is therefore a clear case for shifting up the range of likely fluxes on bright representative pixels from 0.5 to 1 (Langevin et al., 2017) to $0.7-1$, with a median value of 0.85 . With this revised evaluation, the median reflectance factor of large particles collected by COSIMA decreases to $9 \%$ (7.5\%-11\% with error bars), still higher than the geometric albedo inferred by OSIRIS (5-7\% at 645 nm, (Fornasier et al., 2015).

\section{Comparing optical properties at microscopic and macroscopic scales: possible selection biases and scale effects}

The $100 \mu \mathrm{m}$-sided particles for which reflectance properties can be evaluated by COSIMA have been collected at distances of $\sim 10 \mathrm{~km}$ to several $100 \mathrm{~km}$ away from the nucleus, at heliocentric distances from 3.3 AU to perihelion (1.24 AU). There is no clear trend of the median reflectance of COSIMA particles with heliocentric or cometocentric distance (Langevin et al., 2017), which makes it unlikely that optical properties are altered on the way from the nucleus to COSIMA (short transit time: outburst of September 5, 2016).

One cannot exclude a bias at ejection (brighter material than average) or at collection. Dark particles are expected to be constituted of a larger proportion of organic material compared to silicates. They are likely to present a higher porosity/lower mean density, which would make it easier for them to leave the nucleus Skorov and Blum (2012). Recent results from VIRTIS-H (Bockelée-Morvan et al., 2019) show that dust in the coma (expected to be a few $\mu \mathrm{m}$ in size) exhibit a trend towards higher albedo in the near-IR for $\mu \mathrm{m}$-sized particles from a few $\mathrm{km}$ to $10 \mathrm{~km}$ away from the nucleus. This could be linked to a scale effect, with larger particles closer to the nucleus. Only one particle larger than $100 \mu \mathrm{m}$ has been collected by COSIMA closer than $10 \mathrm{~km}$, hence we cannot directly compare reflectance properties in the inner coma with that observed by VIRTIS-H in the near IR at smaller scales. It is however interesting to note that the VIRTIS-H observations were performed at phase angles $\sim 90^{\circ}$, close to that of Cosiscope, in a relatively flat angular range of the dust phase function (Frattin et al., 2019).

Recent results indicate that the collection efficiency of COSIMA is not $100 \%$ even at low speed (Ellerbroek et al., 2017). Particles at the low end of the reflectance range are weaker than bright particles due to a larger contribution from organic material, which should favor collection as "shattered clusters" similar to those observed with Cosiscope. Therefore, possible collection biases would result in a lower mean albedo for COSIMA particles, hence they cannot provide an explanation for the remaining discrepancy between the OSIRIS range (5-7\%) and the best estimate of the COSIMA reflectance factor (9\%).

The most likely interpretation lies in the large difference of scales between these two sets of observations. The OSIRIS IFOV (18.6 $\mu \mathrm{rad})$ corresponds to a pixel size of $37 \mathrm{~cm}$ from $20 \mathrm{~km}$, which is a factor of 20,000 larger than the $14 \mu \mathrm{m}$ pixel size of Cosiscope. As demonstrated by Fig. 4, the cometary surface as observed by OSIRIS with sub-meter resolution is very irregular in many areas. Relatively smooth areas exhibit structure at sub-meter scales. There is very significant self-shadowing by surface irregularities which account for $22 \%$ of the darkening at large phase angles (Vincent, 2019).

The topography of the cometary nucleus is complex at intermediate scales, as shown by images obtained by the Philae panoramic cameras (Bibring et al., 2015) and descent camera (Mottola et al., 2015) after landing at resolutions of a few $\mathrm{mm}$ /pixel, intermediate between that of Cosiscope and that of OSIRIS. The probability of multiple scattering increases with scale, as photons escaping a surface element are likely to hit irregularities at higher scales, which can account for the remaining discrepancy between OSIRIS and COSIMA.

Differences in photometric behavior between single particles and surfaces observed at macroscopic scales are supported by laboratory studies (Shkuratov et al., 2007; Levasseur-Regourd et al., 2019) and OSIRIS observations (Bertini et al., 2017). A photometric model of dust in the coma taking into account polarization (Zubko et al., 2017) leads to the conclusion that scattering properties of particles in a scale range of a few $\mu \mathrm{m}$ (100 times smaller than the large COSIMA particles for which reflectance factors have been evaluated) are underestimated by a factor of 2 or more if macroscopic photometric properties are applied. The results of this model are in line with the observations by VIRTIS-H 


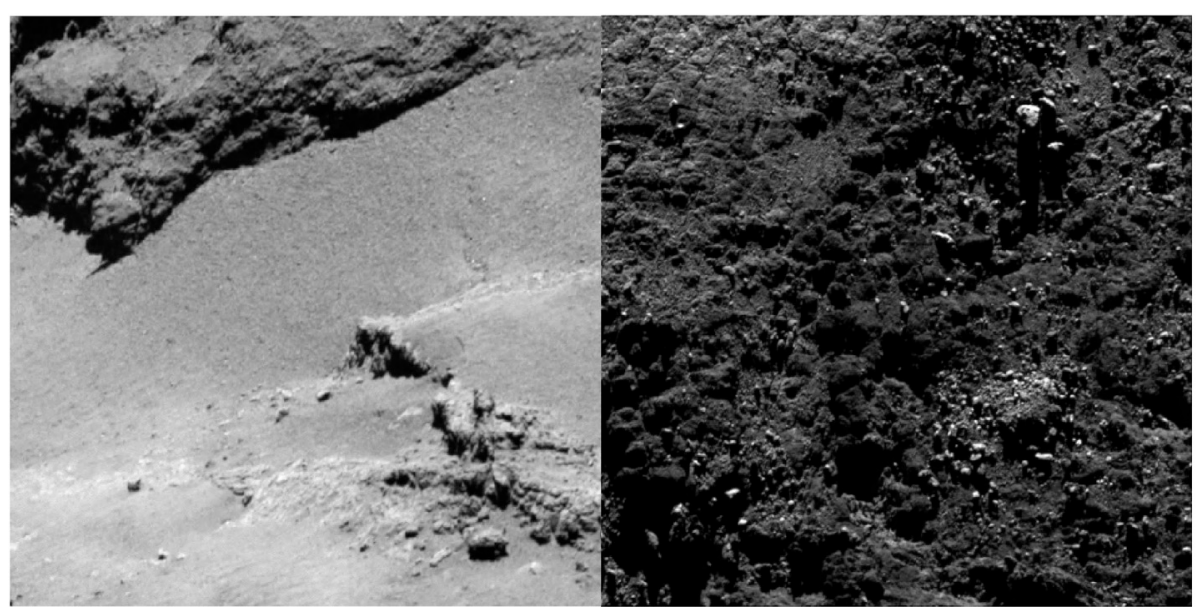

Fig. 4. Two regions $\sim 160 \mathrm{~m}$ across of the nucleus of 67/P Churyumov-Gerasimenko observed by OSIRIS NAC from a distance of $\sim 17 \mathrm{~km}$ at a phase angle of $66^{\circ}$ (left) and close to the terminator (right) with a resolution of $\sim 32 \mathrm{~cm} /$ pixel. Some regions (top of the image on the left) are much rougher at scales larger than $1 \mathrm{~m}$ than others (e.g. the center left region of the image on the left) and even relatively smooth areas show structure when observed close to the terminator. Copyright: ESA/Rosetta/MPS for OSIRIS Team MPS/UPD/LAM/IAA/SSO/INTA/UPM/DASP/IDA CC BY-SA 4.0.

(Bockelée-Morvan et al., 2019) which lead to reflectance factors of 14\% for small dust particles in the coma $10 \mathrm{~km}$ away from the nucleus, a factor of 2 higher than the albedo of the nucleus.

This issue of the size threshold between the photometric properties of single particles and that of surfaces is discussed in (Fulle et al., 2018); which mention that while a precise determination of this threshold from modelling is not yet available, surface scattering properties should not be expected up to a size range of $2.5 \mathrm{~mm}$, well beyond the size range of COSIMA particles (a few $10 \mu \mathrm{m}$ to a few $100 \mu \mathrm{m}$ ). The complex issues related to cometary dust on the nucleus and in the coma are addressed in a recent review article (Levasseur-Regourd et al., 2018).

\section{Conclusions}

Laboratory measurements of the optical properties of "silver black" reference targets at high incidence support the Lambert scattering assumption made in (Langevin et al., 2017). Laboratory measurements have also been performed on two classes of analog particles ( $\mathrm{SiO} 2 \mathrm{ag}$ gregates and Allende meteorite powder), with limitations on the maximum incidence due to low SNR. The observed behavior is also consistent with a Lambert scattering law (albeit with large error bars). Cometary particles collected by COSIMA exhibit a complex substructure. The mean free path of 20-25 $\mu \mathrm{m}$ within aggregate particles (Langevin et al., 2017) is consistent with a high level of porosity, also supported by evaluations of particle strength (Hornung et al., 2016). With such a high porosity, multiple scattering is expected to play a major role, which is consistent with a Lambert scattering law.

The initial evaluation of the uncertainty on the reflectance factor of each particle (factor of 2) can be considered conservative, with the most likely estimate on the low side of this range. This leads to a median value of $9 \%$ for the reflectance factor of COSIMA particles, closer to that from OSIRIS (5-7\%) than the initial evaluation (10.8\%) but still on the bright side after compensating for the different observation geometry.

Selection effects at departure from the nucleus and/or at collection by COSIMA cannot be excluded. However, collection biases are expected to favor the darkest particles as those more likely to be collected. The most likely interpretation of the remaining discrepancy by a factor of 1.5 in terms of photometric properties between COSIMA and OSIRIS is therefore linked to the difference in scale by a factor of $\sim 10,000$, with a higher probability of multiple scattering at larger scales.

The reflectance factors observed by COSIMA provide evidence that the reflectance of cometary material as derived from macroscopic observations is underestimated for cometary dust particles at intermediate scales (a few $10 \mu \mathrm{m}$ to a Skorov and Blum (2012) few $100 \mu \mathrm{m}$ ), albeit by a smaller factor (1.5) than the factor of 2 observed by VIRTIS-H or modeled by (Zubko et al., 2017) for $\mu \mathrm{m}$-sized particles. Cometary dust production rates from remote sensing using dust albedos derived from macroscopic observations of cometary nuclei (e.g. (Fratttin et al., 2017) are therefore likely to be overestimated by a factor ranging from 1.5 to 2 , depending on the relative photometric contributions of $\mu \mathrm{m}$-sized particles and larger particles in the coma.

\section{Data availability}

COSIMA data: https://archives.esac.esa.int/psa/\#!Table\%20Vie $\mathrm{w} / \mathrm{COSIMA}=$ instrument.

OSIRIS data: https://archives.esac.esa.int/psa/\#!Table\%20Vie w/OSIRIS $=$ instrument.

\section{Declaration of competing interest}

The authors declare that they have no known competing financial interests or personal relationships that could have appeared to influence the work reported in this paper.

\section{Acknowledgements}

COSIMA was built by a consortium led by the Max-Planck-Institut für Extraterrestrische Physik, Garching, Germany, in collaboration with:the Laboratoire de Physique et Chimie de l'Environnement et de l'Espace,Orléans, France; the Institut d'Astrophysique Spatiale, CNRS/Université ParisSud, Orsay, France; the Finnish Meteorological Institute, Helsinki, Finland; the Universität Wuppertal, Wuppertal, Germany; von Hoerner und SulgerGmbH, Schwetzingen, Germany; the Universität der Bundeswehr, Neubiberg,Germany; the Institut für Physik, Forschungszentrum Seibersdorf, Seibersdorf,Austria; and the Institut für Weltraumforschung, Österreichische Akademieder Wissenschaften, Graz, Austria; and is led by the Max-Planck-Institut fürSonnensystemforschung, Göttingen, Germany. We acknowledge the support ofthe national funding agencies of Germany (DLR, grant 50 QP 1302), France(CNES), Austria (project FWF P26871-N20), Finland and the ESA TechnicalDirectorate. Rosetta is an ESA mission with contributions from its MemberStates and NASA. R.I. acknowledges support from the Labex Exploration Spatiale des Environnements Planétaire (ESEP; no. 2011LABX-030). S.S. was funded by the Swedish National Space Board (contracts 121/11 and 198/15), the Swedish Research Council (contract 2015-04129).

Datasets of the OSIRIS instrument have been downloaded from the ESA Planetary Science Archive (http://archives.esac.esa.int/psa). The authors acknowledge the Principal Investigator O. Sierks of the OSIRIS instrument on board the Rosetta mission for providing datasets in the archive. 


\section{References}

Bentley, M.S., Schmied, R., Mannel, T., 2016. Aggregate dust particles at comet 67P/ Churyumov-Gerasimenko. Nature 537, 73. https://doi.org/10.1038/nature19091.

Bertini, I., La Forgia, F., Tubiana, C., Güttler, C., Fulle, M., Moreno, F., et al., 2017. The scattering phase function of comet 67P/Churyumov-Gerasimenko coma as seen from the Rosetta/OSIRIS instrument. Mon. Not. R. Astron. Soc. 469 (Suppl. 1_2), S404-S415.

Bibring, J.-P., Langevin, Y., Carter, J., 2015. 67P/Churymov-Gerasimenko surface properties from CIVA panoramic images. Science 349. https://doi.org/10.1126/ science.aab0671.

Bockelée-Morvan, D., Leyrat, C., Erard, S., et al., 2019. VIRTIS-H observations of the dust coma of comet 67P: spectral properties and color temperature variability with phase and elevation. A\&A. https://doi.org/10.1051/0004-6361/201834764 (in press).

Ellerbroek, L.E., Gundlach, B., Landeck, A., et al., 2017. The footprint of cometary dust analogues - I. Laboratory experiments of low-velocity impacts and comparison with Rosetta data. MNRAS 469 (Suppl 2), S204-S216. https://doi.org/10.1093/mnras/ stx1257.

Feller, C., Fornasier, S., Hassemann, P.H., et al., 2016. Decimetre-scaled spectrophotometric properties of the nucleus of comet 67P/ Churyumov-Gerasimenko from OSIRIS observations. MNRAS 462, S287-S303.

Fornasier, S., Hasselmann, P.H., Barucci, M.A., 2015. Spectrophotometric properties of the nucleus of comet 67P/Churyumov-Gerasimenko from the OSIRIS instrument onboard the ROSETTA spacecraft. A\&A 583. https://doi.org/10.1051/0004-6361/ 201525901.

Fratttin, E., Cremonese, G., Simioni, E., et al., 2017. Post-perihelion photometry of dust grains in the coma of 67P Churymov-Gerasimenko. MNRAS 469, S195-S203.

Frattin, E., Munoz, O., Moreno, F., et al., 2019. Experimental phase function and degree of linear polarization of cometary dust analogues. MNRAS 484, 2918-2211.

Fulle, M., Bertini, I., Della Corte, V., 2018. The phase function and density of the dust observed at comet 67P/Churyumov-Gerasimenko. MNRAS 476, 2835-2839. https:// doi.org/10.1093/mnras/sty464.

Groussin, O., Sierks, H., Barbieri, C., et al., 2015. Temporal morphological changes in the Imhotep region of comet 67P/Churyumov-Gerasimenko. A\&A 583 (A36). https:// doi.org/10.1051/0004-6361/201527020.

Güttler, C., Hasselmann, P.H., Li, Y., et al., 2017. Characterization of dust aggregates in the vicinity of the Rosetta spacecraft. MNRAS 469, S312-S320.

Hilchenbach, M., Henning, F., Langevin, Y., 2017. Mechanical and electrostatic experiments with dust particles collected in the inner coma of comet 67P by COSIMA on-board Rosetta. Philos. Trans. R. Soc. 375, 2097. https://doi.org/10.1098/ rsta.2016.0255.

Hornung, K., et al., 2014. Collecting cometary dust particles on metal blacks with the COSIMA instrument onboard Rosetta. Planet. Space Sci. 103, 319. https://doi.org/ 10.1016/j.pss.2014.08.011.

Hornung, K., Merouane, S., Hilchenbach, M., 2016. A first assessment of the strength of cometary particles collected in-situ by the COSIMA instrument onboard ROSETTA. Planet. Space Sci. 133, 63. https://doi.org/10.1016/j.pss.2016.07.003.
Kissel, J., Altwegg, K., Clark, B.C., 2007. COSIMA - high resolution time-of-flight secondary ion mass spectrometer for the analysis of cometary dust particles onboard Rosetta. Space Sci. Rev. 128, 823-867.

Langevin, Y., Hilchenbach, M., Ligier, N., 2016. Typology of dust particles collected by the COSIMA mass spectrometer in the inner coma of 67P/Churyumov-Gerasimenko. Icarus 271, 76. https://doi.org/10.1016/j.icarus.2016.01.027.

Langevin, Y., Hilchenbach, M., Vincendon, M., 2017. Optical properties of cometary particles collected by the COSIMA mass spectrometer on-board Rosetta during the rendez-vous phase around comet 67P/Churyumov-Gerasimenko. MNRAS 469, S535. https://doi.org/10.1093/mnras/stx2070.

Levasseur-Regourd, A.C., Agarwal, J., Cottin, H., Engrand, C., Flynn, G., Fulle, M., Gombosi, T., Langevin, Y., Lasue, J., Mannel, T., Merouane, S., Poch, O., Thomas, N., Westphal, A., 2018. Cometary dust. Space Sci. Rev. 214 https://doi.org/10.1007/ s11214-018-0496-3.

Levasseur-Regourd, A.-C., Renard, J.B., Hadamcik, E., Lasue, J., Bertini, I., Fulle, M., 2019. Interpretation through experimental simulations of phase functions revealed by Rosetta in 67P/Churyumov-Gerasimenko dust coma. Astron. Astrophys. 1-6 https://doi.org/10.1051/0004-6361/201834894.

Merouane, S., Zapriduin, B., Stenzel, O., et al., 2017. Evolution of the physical properties of dust and cometary dust activity from 67P/Churyumov-Gerasimenko measured in situ by Rosetta/COSIMA. MNRAS 469, S459-S474. https://doi.org/10.1093/mnras/ stx2018.

Moosmüller, H., Engelbrecht, J.P., Skiba, M., et al., 2012. Single scattering albedo of fine mineral dust aerosols controlled by iron concentration. J. Geophys. Res. 117, D11210.

Mottola, S., Arnold, G., Grothues, H., 2015. The structure of the regolith on 67P/Churyumov-Gerasimenko from ROLIS descent imaging. Science 349. https://doi.org/ 10.1126/science.aab0232.

Shkuratov, Y., Bondarenko, S., Kaydash, V., Videen, G., Muñoz, O., Volten, H., 2007. Photometry and polarimetry of particulate surfaces and aerosol particles over a wide range of phase angles. J. Quant. Spectrosc. Radiat. Transf. 106 (1-3), 487-508. https://doi.org/10.1016/j.jqsrt.2007.01.031.

Skorov, Y., Blum, J., 2012. Dust release and tensile strength of the non-volatile layer of cometary nuclei. Icarus 221, 1-11. https://doi.org/10.1016/j.icarus.2012.01.012.

Vincendon, M., Langevin, Y., Poulet, F., 2007. Recovery of surface reflectance spectra and evaluation of the optical depth of aersols in the neazr-IR using a Monte-Carlo approach : application to the OMEGA observations of high latitude regions of Mars. J. Geophys. Res. 112 https://doi.org/10.1029/2006JE002845. E08S13, 4.

Vincent, J.B., 2019. Cometary topography and phase darkening. A\&A 2019 (in press).

Wolff, M.J., Code, A.D., Groth Edward, J., 1999. Hubble Space Telescope observations of the Martian aphelion cloud belt prior to the Pathfinder mission: seasonal and interannual variations. J. Geophys. Res. 104, 9027-9042.

Wolff, M.J., Smith, M.D., Clancy, R.T., 2009. Wavelength dependence of dust aerosol single scattering albedo as observed by the Compact Reconnaissance Imaging Spectrometer. J. Geophys. Res. 114 https://doi.org/10.1029/2009JE003350. CiteID E00D0.

Zubko, E., Videen, G., Shkuratov, Y., Hines, D.C., 2017. On the reflectance of dust in comets. J. Quant. Spectrosc. Radiat. Transf. https://doi.org/10.1016/ j.jqsrt.2017.07.026. 